\title{
GENOMICS AND EPIGENOMICS IN MAIZE HYBRID KERNEL
}
M. M. Elsahookie
M. J. Al-Khafaji
A. A. Dawood
Dept. of Field Crops Sciences, Coll. of

\section{Review Article}

\section{ABSTRACT}

The endosperm in cereals supplies nutrients to the developing kernel and seedling, and it is the primary tissue that gene imprinting occurs. Developing maize (Zea mays L.) endosperms were analysed for allelic gene expression in both reciprocal crosses of inbreds B73 and Mo17. A high-throughput transcriptome sequencing in kernels at 0,3 up to 15 DAP of both reciprocals were performed, and found a gradual increased paternal transcript expression in 3 and 5 DAP kernels. Meanwhile, in 7 DAP endosperm, most of genes tested gave the ratio 2:1 maternal: paternal, suggesting that paternal genes are almost fully activated at 7 DAP. There were 300 PEGs and 499 MEGs identified across endosperm development stages. A 63 genes out of 116, 234 exhibited parent-specific expression were identified at 7,10 and 15 DAP. Most of paternally expressed genes was at 7 DAP due to deviation of paternal alleles expression at this stage of development. Imprinted genes in terms of relative expression of maternal and paternal alleles differed at least five folds in both crosses. A total of $179(1.6 \%)$ protein coding genes expressed in the endosperm were imprinted, 68 of them showed maternal preferential expression and 111 paternal expression, besides 38 long noncoding RNA were found imprinted and transcribed in either sense or antisense direction from intronic regions of normal protein coding genes or from intergenic regions. Imprinted genes showed clustering around the genome. A total of 21 imprinted genes in the maize hybrid endosperm had differentially methylated regions (DMRs). All DMRs were found to be hypomethylated in maternal alleles and hypermethylated in paternal alleles. These results confirm a complex mechanism controlling endosperm in maize in imprinting, auxin activity, and development regulation. Studying $F_{2}$ kernels on $F_{1}$ plants may shed a new light on controlling kernel number weight in unit of area.

Key words: PEG and MEG, paternal and maternal expressed genes, imprinting, triploid endosperm, DNA methylatiom.

\begin{tabular}{|c|c|}
\hline & محلة العلوم الزراعية العراقية -967-960:69:2018:49) \\
\hline
\end{tabular}

$$
\text { الجينوم وفوق الجينوم في سويداء هجن الذرة الصفراء }
$$

$$
\begin{aligned}
& \text { عبد الباسط عبد الرزلق داود } \\
& \text { وزارة الزراعة }
\end{aligned}
$$$$
\text { مصطفى جمال الخفاجي }
$$$$
\text { مدرس }
$$

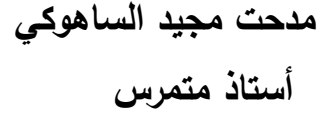

أستاذ متمرس

$$
\text { قسم المحاصيل الحقلية - كلية الزراعة - جامعة بغداد }
$$

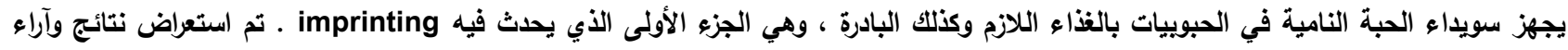

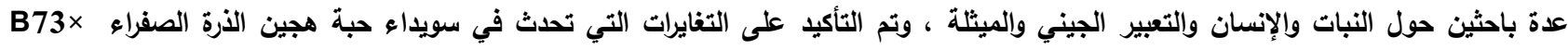

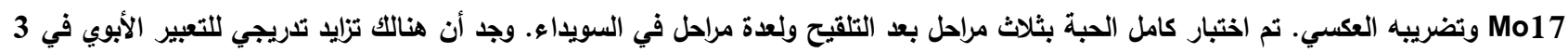

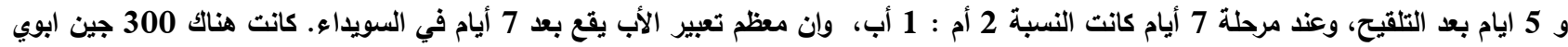

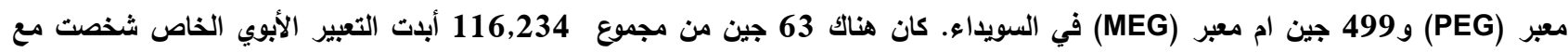

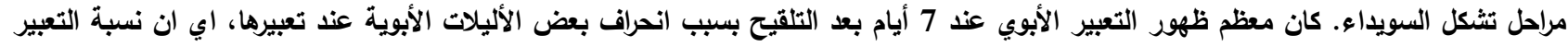

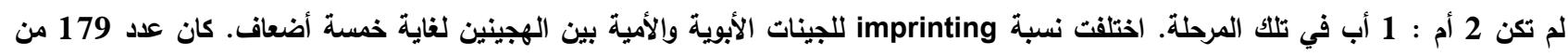

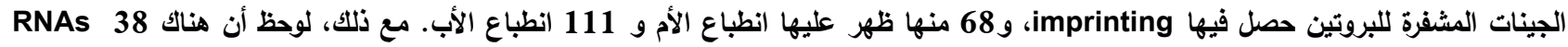

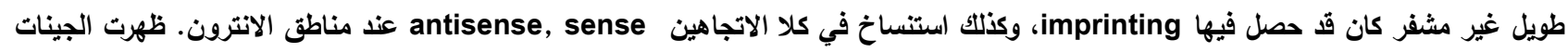

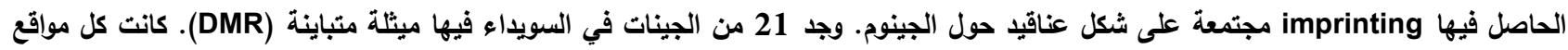

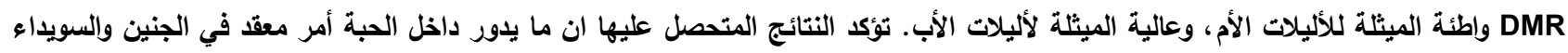

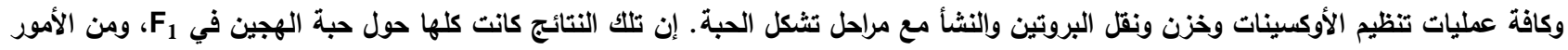

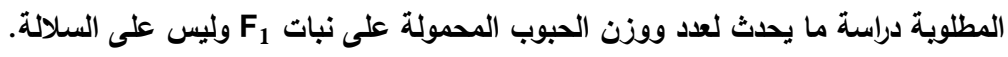

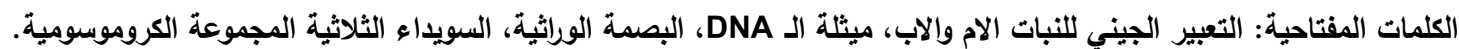




\section{INTRODUCTION}

Hybrids in crop plants are being widely used in world agriculture, for their higher yield and/ or quality. New maize hybrids are higher in grain yield than old hybrids by $1.3-3.1 \mathrm{t} \mathrm{ha}^{-1}$ (8). Kernel weight in newer hybrids have $15 \%$ - $23 \%$ increase and an average of 150 kernels per square meter as compared to old hybrids (10). Tollenaar and Lee(37) estimated that the annual increase in maize grain yield in the U.S is about $118 \mathrm{~kg} \mathrm{ha}^{-1}$, and about $65 \%$ of that increase is due to genetic improvement. There are several explanations of hybrid vigour in crop plants according to genomic influence, but epigenomics have been shown to play an important role in kernel weight and embryo. Kumar (26) reported that epigenome is referred to the biochemical changes in nuclease DNA, post-translational modifications in histone proteins, and variations in the biogenesis of small noncoding RNAs in the cell. These changes are often responsible for variation in gene expression without a change in the base pairs of nucleotide sequence. Changes due to epigenetics are commonly found in gene regulation through developmental stages, tissue differentiation, and suppression of transposable elements in high living beings, and that epialleles were functioning $(2,9,11$, $16,22,26,38)$. Epigenomic studies on human are more than those on plants, especially those applied on cancer and brain disorders in human (16), meanwhile, Bressman and Zhu (7) reported that total articles published on epigenetic in two years was about 14,000 . Epigenomic changes are in many cases subjected to influence of environmental stimuli affecting plasticity of the plant in that environment. Some of these changes could disappear after removal of that environmental influence, but sometime it is inherited from generation to generation $(14,15,25,26)$. In plant evolution, most of the changes are due to changes in DNA nucleotide sequence, but epigenomic could play a role in plant evolution. The best example of epigenomic rapid changes is that in case of human diseases, especially cancer, and some of neurological developments.

\section{Mechanisms of epigenomics}

Genes and transposons can exist in variable DNA methylation states, with potentially differential transcription. Novel non parental and heritable epialleles arise at many genic loci in the hybrid (32). This suggest that combining distinct epigenomes of two parents can create novel patterns of genes and transposons regulation. Lauss et al (27) reported that genomic explanations were not sufficiently explain or predict hybrid vigour. Meanwhile, Groszmann et al $(18,19)$ believe that there is a growing evidence that epigenics play a role in hybrid vigour in several crop species. Jones (24) stated that about $4 \%$ of human cytosine is methylated and there are three contexts of cytosine; $\mathrm{CG}, \mathrm{CHG}$, and $\mathrm{CHH}$, when $\mathrm{H}=\mathrm{A}$, T, or C. However, Slotkin et al (36) reported that more than $80 \%$ of the cytosine in CG context is methylated in human genome, but in plants, $\mathrm{CG}$ and $\mathrm{CHG}$ methylation is maintained by methyltransferase 1 and chromomethylase 3 , respectively, while $\mathrm{CHH}$ methylation is maintained by RdDM (RNA-dependent DNA methylation) or the chromatin remodeler DDM1. Meanwhile, methylaition at non-CG sites plays a role in plants by silencing the activity of foreign DNA by RdDM pathway (28). Specific mechanisms are required to make and / or maintain the specific regions free of methylation by DNA demethylation processes which may take place by the active or passive method. The active DNA methylation requires enzymatic glycosylases including DME, DML2, and DML3 in plants (48).

\section{Chromatin structure and genome stability}

Plants of eukaryotes have DNA tightly packaged in a chromatin structure of nucleosomes, the later is composed of protein octamer consisting of pairs of histones $\mathrm{H}_{2} \mathrm{~A}$, $\mathrm{H}_{2} \mathrm{~B}, \mathrm{H}_{3}$, and $\mathrm{H}_{4}$ (26). Histone modification has been reported to be associated with repression or activation of genome regions depending on the level of methylation of the amino acid residue affected, which is dynamically regulated by the actions of histone methylatransferases and histone demethylases. Bhaumik et al (4) reported that some of the well-known core histone modifications include methylation of Lys and 
Ard, acetylation of Lys, phosphorylation of Ser and Thr, and mono- or poly-ubiquitylation of Lys. However, these post-translational modifications can take place or removed by protein modifiers, such as; histonemethyltransferases - demethylases, acetyltransferases and -deacetylases (26). Chromatin modifications are affected by different biotic and / or a biotic stresses, and that could be reflected on gene expression of the living being $(14,15)$. Chromatin structure could be different in compactness due to epigenetic mechanisms occur due to environmental variables (35). These changes could be inherited to the next progeny or disappear according to magnitude of epigenetic effect in the phenotype of that organism. There is evidence that chromatin modifications and RNAs are involved in controlling gene expression at transfcriptional as well as post-transcriptional level and these changes could be reversible depending on degree of activation (43). Plants nuclear genomes may contain more than 50\% methylcytosine in all the three nucleotide contexts (46). RNAi silencing and knockout mutation of stress inducible histone deacetylase in maize and Arabidopsis resulted in increased histone acetylation leading to the depression of silenced genes. Cytosine methylation further strengthens the histone modification patterns contributing to gene silencing (33).

\section{Inheritance of epigenetic mark}

There are epigenetic changes occur under some stress variables in situ or in vivo or in vitro. However, some of these changes are inherited to the next generation, while some others disappear. Zheng et al (47) reported that drought adaptability of rice plant has been improved, because of multi-generational drought exposure. They identified appearance of some drought induced epimutations could maintain the altered DNA methylation level in the subsequent generations. Their results suggest that epigenetic mechanisms play important role in plant adaptation and / or evolution, at least in some specific conditions, such as salinity stress and drought stress. Elsahookie (14) reported that plants selected under salinity or drought stress showed a level of tolerance to that stress in next generations not known before in the original cultivar plants.

\section{Epigenetics in hybrid maize kernels}

Because of the high importance of epigenetics in plants and humans, several researchers have worked out different approaches in this growing new science. Some of them have worked on Arabidopsis, Oryza , and Brassica $(18,21,31,32,34,43)$. Other researchers their concern was on some human diseases $(2,4,9$, 11, 16). Meanwhile, studies on DNA methylation in plants under stress were undertaken by some researchers $(3,6,7,20$, $22,23,24,25,26,28,30,36,38,41)$. However, our most important part of this review will be on DNA methylation in maize hybrid kernel which have been studied by many researchers, bu't we have focused our concern on those studies of extent coverage (1, $5,8,10,12,13,17,19,27,29,33,37,39,40$, 41, 42, 43, 45).

\section{Kernel development in cereals}

The development of kernels in angiosperms in general, and in cereals per se, counts on activities of both embryo and endosperm gene regulations. If we take the embryo, as it is diploid (2n), it has effects of 1 maternal m: 1 paternal $p$, whereas the endosperm which is triploid (3n), the ratio will be $2 \mathrm{~m}: 1 \mathrm{p}$. That is due to double fertilization of the endosperm, in which $2 n$ from central cells and $1 \mathrm{n}$ from sperm nucleous (pollen grain). Hence, studying the effects of epigenetic on kernel is helpful to understand the consequences of gene activities through the kernel stages of development. Sabilli and Larkins (34) stated that in cereals, immediately following cellularization, the endosperm undergoes an intense period of cell division, followed by differentiation and specialization to from a nutritive tissue that supports the embryo and the developing seedling. This mitotic phase in maize begins in the central region at 4-5 DAP (days after pollination) and lasts until 8 to $12 \mathrm{DAP}$, but it may continue until 20 to 25 DAP in the peripheral regions of the endosperm. Differentiation of the endosperm cells follows these processes and result in four primary regions or tissues; aleurone, starchy endosperm, transfer layer, and the embryo surrounding region. Elsahookie (13) and Yousif and Elsahookie (42) found that in all 
crosses of inbreds, the female controls kernel traits such as weight, shape and colour, except when source pollen is saccharata.

\section{Results obtained on maize inbreds (B73×Mo17):}

\section{1-Base pairs of Mo17 and B73}

Xin et al (40) obtained results on genome of maize inbred Mo17, that its genome was $2,058,527,894$ bases containing a total of $117,847,390$ bp short gaps. Thus, the coverage of the Mo17 genome was $\sim 94.3 \%$ producing a similar genome size (of inbred B73) 2,066,432,971 bp. The identification of single nucleotide polymorphism (SNPs) and insertions, and deletions was performed, and found that there were 6,557,611 SNPs indentified 157,994 insertions, and 191,549 deletions between inbreds B73 and Mo17 genomes.

\section{2-Paternal transcriptome in the reciprocals}

Higher numbers and transcripts abundances of genes with parental alleles in the cross Mo17 $\times$ B73 were found than in the reciprocal B73 $\times$ Mo17 at 3 and 5 DAP, namely at 3 DAP, 1194 genes with activated paternal alleles were indentified in the cross Mo17×B73 compared with 874 genes in B73 $\times$ Mo17 cross (40). Meanwhile, at 5 DAP, 4295 genes were parentally activated in the cross Mo17 $\times$ B73 compared with 3478 in B $73 \times$ Mo17 cross. One of the surprising thing they found, is that only 2561 of these genes were commonly activated, while in 7 DAP, 10 DAP and 15 DAP endosperm, the proportion of commonly activated genes accounted for $74.3 \%, 71.3 \%$ and $70.9 \%$ respectively. Then they compared paternally activated genes in the 3 and 5 DAP kernels of the Mo17×B73 and $\mathrm{B} 73 \times \mathrm{Mo} 17$ crosses, and found that was of similar activation.

\section{3-SNPs in maize hybrid endosperm}

Xin et al (40) have analyzed the whole kernel imprinted genes, and found 11,027 and 10,573 imprinted genes, and 7777 SNPs containing genes, found in the 7,10 and 15 DAP endosperm, respectively. The genes exhibiting parent-specific patterns in both reciprocal crosses were selected as candidate imprinted genes, and found that 284,606, and 190 genes were determined to have allele-biased, parentof- origin specific expression patterns at 7, 10 and 15 DAP, respectively. There were 300
PEGs and 499 MEGs identified from the three developing endosperm stages. Among the 499 MEGs, 418 were identified at 10 DAP only, and only 15 were indentified at all three time points. Meanwhile, among the 300 PEGs, 213, 130 , and 163 were observed at 7,10 and 15 DAP respectively.

\section{4-Nonimprinted genes in the endosperm}

Nonimprinted allele-specific expression is widespread and believed to be responsible for quantitative variations in the phenotypic traits of hybrid offspring (40). In the three stage samples of endosperm, 1688 B73 allelespecific and 1130 Mo17 allele-specific genes were detected, that exhibited expression dosages deviating from the $2 \mathrm{~m}: 1 \mathrm{p}$ ratio. Among these, 303 B73 genes and 128 Mo17 genes exhibited a non-parent-specific, monoallelic expression pattern in both reciprocal crosses. The second type of genes are inbred line-dependent imprinted gene that exhibits allele-specific expression in one direction of each reciprocal pair of crosses, but biallelic expression in the other direction (39).

\section{5-MEGs and PEGs expression in the endosperm}

Four groups out of the 290 imprinted genes were identified (40). These were; regulation of gene expression by genetic imprinting, endosperm development, response to hormone stimulus, and DNA-binding. These functional categories indicated involvement of the imprinted genes in hormone signaling pathways, and transcriptional regulation of endosperm development. When the 194 MEGs and 96 PEGs were examined separately, they found only two categories and one category exhibiting significant enrichments for MEGs and PEGs, respectively. The first category of MEGs was the response to hormone stimulus which included 13 genes, 6 of which were involved in the auxin-mediated transcriptional response. The second was the cytoplasmic membrane bounded vesicle, which included 26 genes encoding a variety of enzymes, transported proteins, and cell wall formation proteins located in the cytoplasmic membrane , suggesting that these MEGs might be involved in intercellular nutrient transport and signal transduction. The only one enriched among the 29 PEGs was the binding category. This category represented various forms of 
molecular interactions, such as, DNA binding, RNA binding, protein binding, and ATP binding. The functions of MEGs and PEGs are not known.

\section{6-Pattern of MEGs and PEGs imprinting}

MEGs and PEGs found in maize hybrid endosperm exhibited different patterns of gene imprinting during endosperm stages of development (40). They observed PEGs predominately detected at DAP and MEGs predominately detected at 10 DAP. Out of the 194 MEGs identified in the three stages, 150 were shown to be expressed maternally in both crosses uniquely at 10 DAP. On the other hand, the 7 DAP endosperm contained the highest number of PEGs, 26 of which were shown to be paternally expressed only at this developmental stage in both crosses. However, they found the occurrence of 7 DAP specific PEGs were mainly due to the significantly deviated paternal allele ratio, whereas the 10 DAP specific MEGs could be primarily attributed to the sharply increased expression levels at the respective stages. Three 7 DAP specific PEGs and nine 10 DAP specific MEGs were studied, all the three PEGs exhibited paternally biased expression patterns at 7 DAP in both reciprocal crosses, although their maternal alleles were strongly expressed in Mo17×B73 cross. All nine MEGs tested, showed a predominant pattern of expression at 7 or 15 DAP in both reciprocal crosses. Among the 10 DAP specific MEGs, two were identified to encode auxin response factors of the auxin / indole-3-acetic acid family, suggesting that endosperm nutrient uptake from maternal tissue is maternally controlled.

\section{7-Paternal genome in the 7 DAP endosperm}

The available knowledge of the timing and extent of activity of maternal and paternal genomes after fertilization is limited. Nodine and Bartel (30) reported that results obtained on maize kernels suggested that the activation of paternal and maternal alleles could be relatively delayed in the embryo, although contrary evidence indicates that the two sets of parented alleles are activated simultaneously. Xin et al (40) found that at 3 DAP, 941 genes were shown to produce paternal transcripts less than the maternal transcripts. Of the 941 genes, 923 were also expressed at zero DAP suggesting the apparent expression bias may not necessarily be a reflection of a delay in paternal genome activation, but rather due to expression or presence of the transcripts in the maternal kernel tissues. So, it is reasonable to conclude that paternal genome activation was nearly fully achieved by 7 DAP or earlier, because the majority of the 11,027 genes with SNPs were shown to be biallelically expressed, and the proportion of the expressed genes with the expected $2 \mathrm{~m}: 1 \mathrm{p}$ ratio was similar in the 7 DAP and 10 DAP endosperms.

\section{8-DNA methylation and imprinting of MEGs and PEGs}

Zhang et al (45) have done an extensive work on maize hybrid kernels. They used DNA from 12 DAP endosperm of the B73 $\times$ Mo17 hybrid, and obtained 866 million 100-bp paired-end reads. To be analyzed for potential allelespecific methylation, a gene required sufficient read coverage for both maternal and paternal genomic alleles in regions with at least one SNP between B73 and Mo17. A total of 98 genes (39 MEGs, 59 PEGs) met this criteria. Of these genes, 9 MEGs and 8 PEGs showed differential $\mathrm{CpG}$ methylation between the two parental alleles. They found 4 DMRs for 4 noncoding RNAs among 13 analyzable transcripts. On the other hand, they reported that at least 699 genes are potentially imprinted in maize endosperm along with 111 PEGs and 68 MEGs, and 38 long noncoding RNAs. They believed that these noncoding RNAs have a regulatory functions in maize genome. DNA methylation has long been regarded as a key player in epigenetic regulation. Differential methylation has been shown for several known imprinted genes between the maternal and paternal alleles. These results showed that only $17.3 \%$ of the maize imprinted genes have differential methylation between the two parental alleles. The large number of imprinted genes showing no differential methylation between the parental alleles suggest that methylation is only involved in the regulation of a small portion of imprinted genes. Both MEGs and PEGs having DMRs showed a pattern of maternal hypomethylation and paternal hypermethylation. According to the results obtained, the large number of imprinted protein-coding genes, long noncoding RNAs, and their clustered distribution identified 
clearly, demonstrate complex parent-of-origindependent epigenetic regulation throughout maize endosperm development. Inoue et al (23) revealed that a few genomic loci are maternally imprinted because of the inheritance of maternal histone 3 lysine 27 trimethylation (H3K27me3). Meanwhile, Brannan and Bartolomei stated that the imprinted genes in human were shown to fall into clusters in the genome. Accordingly, Dong et al (12) stated that DNA methylation plays an important role in the allele-specific maternal expression of imprinted genes (MEGs) and paternally expressed imprinted genes (PEGs) are shown to be regulated by the DNA methylation glycosylase and / or DNA methyltransferase. However, many imprinted genes are associated with the differentially methylated regions (DMRs) where the maternal alleles are hypomethylated and the paternal alleles are hypermethylated. Meanwhile, histone modifications represent another level of epigenetic modifications. Genome-wide analyses have shown that many types of histone modifications are associated with the expression or repression of genes in many plants. They also found an allele specific profiles of $\mathrm{H} 3 \mathrm{~K} 4 \mathrm{me} 3 \mathrm{and} \mathrm{H} 3 \mathrm{~K} 36 \mathrm{me} 3$ modifications on a genome-wide scale in maize endosperm at $12 \mathrm{DAP}$, and that was associated with both imprinted protein- coding genes and imprinted noncoding RNAs. They concluded that allele-specific active histone modifications (H3K4me3and H3K36me3) with allel-specific DNA methylation, and repressive modification (H3K27me3) provided a unique look of the regulation of genomic imprinting in maize. When they used histone $\mathrm{H}_{3}$ lysine 4 trimethylation ( $\left.\mathrm{H} 3 \mathrm{~K} 4 \mathrm{me} 3\right)$ and lysine 36 trimethylation (H3K36me3) antibodies, $\mathrm{H} 3 \mathrm{~K} 4 \mathrm{me} 3$ modifications were generally restricted to the transcription start sites, and H3K36me3 modifications were accumulated to high levels in gene bodies and both H3K4me3 and H3K36me3 showed positive correlation with transcription levels in maize endosperm. Their results on B73 and Mo17 reciprocal crosses showed 1393 H3K4me3 peaks and $980 \mathrm{H} 3 \mathrm{~K} 36 \mathrm{me} 3$ peaks in both reciprocal crosses endosperms. According to the relationship between imprinted genes and the allele-specific
H3K4me3 and H3K36me3 peaks, they used samples isolated from 12 DAP hybrid endosperm tissues from both reciprocal crosses, and a total of 54 MEGs and 90 PEGs were identified. That was when the expression level of active alleles at least five times than of silenced alleles. According to the results obtained in the forth going review, the genome-wide studies on plant in general and hybrid maize endosperms became clear in mechanism of gene action, imprinting and modifications. These things were changing with changing stage of days after pollination. Therefore, epigenomics are the second way to modify DNA and gene expression. Some of these modifications are inherited to next lineages, while some of them are not. Epigenomics are likely to occur more under a biotic or biotic stresses; such as salinity, drought and extreme temperatures and auxins used in tissue culture. Selection of desirable phenotypes could survive well under a remarkable merit of selection. Highthroughput techniques being used today have proved to be very helpful to clarify routes of epigenetic mechanisms in plants and humans. The last text could be highly beneficial when scientists get better techniques to look for the functions of I-motif discovered later in human cell chromosomes, that form in cytosine rich regions of the genome(44).

\section{REFERENCES}

1. Barber, W., W. Zhang, H. Win, K. Varala, J. Dorweiler, M. Hudson and S. Moose. 2002. Repeat associated small RNAs vary among parents and following hybridization in maize. PNAS, 109(26): 10443 - 10449

2. Behera, P. 2017. Epigenetic changes in breast cancer: current aspects in India. J. Bioengineer Biomedical Sci., 7:223 - 245

3. Benayoun, B., E. Pollina, and A. Brunet. 2015. Epigenetic regulation of ageing: linking environmental inputs to genomic stability. Nat. Rev. Mol. Cell Biol., 16:593 - 610

4. Bhaumik, S., E. Smith, and A. Shilatifard. 2007. Covalent modifications of histones during development and disease pathogenesis. Nat. Struct. Mol. Biol., 14:1008 - 1016

5. Birchler, J., H. Yao, S. Chadalyandi, D. Vaiman and R. Veitia. 2010. Heterosis. Plant Cell, 22:2105 - 2112 
6. Brannen, C., and M. Bartolomei. 1999. Mechanisms of genomic imprinting. Curr. Opin. Genet. Dev., 9:164 - 170.

7. Bressman, R., and K. Zhu. 2014. Epigenetic connects the genome to its environment. Plant Breeding Rev., 37:69 - 141

8. Chen, K., S. Kumudini, M. tollenaar, and T. Vyn. 2015. Plant biomass and nitrogen partitioning changes between silking and maturity in newer versus old maize hybrids. Field Crops Res., 183:315 - 328

9. Chen, K., J. Zhang, Z. Guo, Q. Ma, Z. Xu, and Y. Zhou. 2016. 5- hydroxymethylcytosine is linked to gene body hypermethylation in kidney cancer. Cell Res., 26: $103-118$

10. Chen, K., J. Camberato, and T. Vyn. 2017. Maize grain yield and kernel component relationship to morphophysiological traits in commercial hybrids separated by four decades. Crop Sc., 57: 1 - 17

11. Delgado-Morales, R., R. Agis-Balboa, M. Esteller, and M. Berdasco. 2017. Epigenetic mechanisms during ageing and neurogenesis as novel therapeutic avenues in human brain disorders. Clinical Epigenetics, 9:67 - 73

12. Dong, X., M. Zhang, J. Chen, L. Ping, N. Zhang, X. Wang and J. Lai. 2016. Dynamic and antagonistic allele-specific epigenetic modifications controlling the expression of imprinted genes in maize endosperm. Molecular Plant, 10: 442 - 455

13. Elsahookie, M. M. 2005. Xenia or hybrid vigour in maize subspecies crosses. TIJAS, 36(6): $57-62$

14. Elsahookie, M. M. 2013. Epigenetics in Plants and Animals. Univ. of Baghdad, Coll. of Agric., pp. 121 (in Arabic).

15. Elsahookie, M. M. 2014. Number of genes interactive models in plants grown under stress variables. TJAS, 45(1): $99-104$

16. Farias, N., N. Ho, S. Butler, L. Delaney, J. Morrison, and S. Shahzad. 2015. The effects of folic acid on global DNA methylation and colonsphere formation in colon cancer cell lines. J. Nutr. Biochem., 26: 818 - 826

17. Grimanelli, D., E. Perotti, J. Ramrez, and O. Leblanc. 2005. Timing of the maternal-tozygotic transition during early seed development in maize. Plant Cell, 17: 1061 1072

18. Groszmann, M., I. Greaves, Z. Albertyn, G. Scofield, W. Peacock, and E. Dennis. 2011.
Changes in 24-nt si RNA levels in Arabidopsis hybrids suggest an epigenetic contribution to hybrid vigour. PNAS, 108(6): 2617 - 2622

19. Groszmann, M., I. Greaves, R. Fujimoto, W. Peacock, and E. Dennis. 2013. The role of epigenetics in hybrid vigour. Trends Genet., 29: $684-690$

20. Gutierez-Marcos, J., and H. Dickinson. 2012. Epigenetic reprogramming in plant reproductive lineages. Plant Cell Physiol., 53(95): $817-823$

21. Ha, M., J. Lu, L. Tian, V. Ramachandran, K. Kasschau, E. Chapman, J. Carington, X. Chen, X-J, and Z. Chen. 2009. Small RNAs serve as a genetic buffer against genomic shock in Arabidopsis interspecific hybrids and allopolyploids. PNAS, 106(42): 17835 17840

22. Hanna, C. and G. Kelsey. 2017. Genomic imprinting beyond DNA Methylayion: a role for maternal histones. Genome Biol., 18: 177 180.

23. Inoue, A., L. Jiang, T. Suzuki, and Y. Zhang. 2017. Maternal H3K27me3 controls DNA methylation-independent imprinting. Nature, 547(7664): $419-424$

24. Jones, P. A. 2012. Functions of DNA methylation: islands, start sites, gene bodies and beyond. Nat. Rev. Genet., 13: $484-492$

25. Kumar, S. and A. Singh. 2016. Epigenetic regulation of abiotic stress tolerance in plants.Adv. Plants Agric. Res., 5:5,00179. Doi:10.15406/apar.2016.05.00179

26. Kumar, S. 2017. Epigenetic of plant responses to environmental stress. Doi:10.20944/preprints

27. Lauss, K. R. Wardinaar, R. Oka, M. Van Hulten, V. Guryev, J. Keurentjes, M. Stam, and F. Johannes. 2018. Parental DNA methylation states are associated with heterosis in epigenetic hybrids. Plant Physiol., 176: $1627-1645$

28. Law, J., and S. Jacobsen. 2010. Establishing, maintaining and modifying DNA methylation patterns in plants and animals. Nat. Rev. Genet., 11: $204-220$

29. Liu, H., L. Ma, X. Yang, L. Zhang, X. Zeng, S. Xie, H. Peng, S. Gao, H. Lin, G. Pan, Y. Wu, and Y. Shen. 2017. Integrative analysis of DNA methylation, mRNAs, and amall RNAs during maize embryo dedifferentiation. BMC Plant Biol., 17: 105 - 116 
30. Nodine, M., and D. Bartel. 2012. Maternal and paternal genomes contribute equally to the transcriptome of early plant embryos. Nature, 482: $94-97$

31. Radoev, M., H. Becker, and W. Ecke. 2008. Genetic analysis of heterosis for yield and yield components in rapeseed (Brassica napus L.) by quantitative trait locus mapping. Genetics, 179: 1547 - 1558

32. Rigal, M., C. Becker, T. Pelissier, R. Pogprelcnik, J. Devos, Y. Ikeda, D. Weigel, and O. Mathieu. 2016. Epigenome confrontation triggers immediate reprogramming of DNA methylation and transposon silencing in Arabidopsis thaliana $F_{1}$ hybrids.

PNAS, www.pnas.org/cgi/doi/10.1073/pnas.16 $\underline{00672113}$

33. Rossi, V., S. Locatelli, S. Voroto, G. Don, R. Pirona, D. Henderson, H. Hartings, and M. Motta. 2007. Maize histone deacetylase hda 101 is involved in plant development, gene transcription, and sequence-specific modulation of histone modification of genes and repeats. Plant Cell, 19: 1145 - 1162

34. Sabilli, P., and B. Larkins. 2009. The development of endosperm in grasses. Plant Physiol., 149: $14-26$

35. Seong, K., D. Li, H. Shimizu, R. Nakamura, and S. Ishii. 2011. Inheritance of stress-induced, ATF-2 epigenetic change. Cell, 145: $1049-1061$.

36. Slotkin, R., M. Vaughn, F. Borges, M. Tanurdzic, J. Becker, J. Feijo, and R. Martienssen. 2004. Epigenetic reprogramming and small RNA silencing of transposable elements in pollen. Cell, 136: $461-472$

37. Tollenaar, M., and E. Lee. 2011. Strategies for enhancing grain yield in maize. Plant Breeding Rev. 34: 37 - 82

38. Wan, J., V. Oliver, G. Wang, H. Zhu, D. Zack, and S. Merbs. 2015. Characterization of tissue-specific differential DNA methylation suggests distinct modes of positive and negative gene expression regulation. BMC Genome, 16: 49 - 58

39. Waters, A., I. Makarevitch, S. Eichten, R. Swanson-Wagner, C. Yeh, W. $\mathrm{Xu}, \mathrm{P}$. Schnable, M. Vaughn, M. Gehring, and N.
Springer. 2011. Parent-of-origin effects on gene expression and DNA methylation in the maize endosperm. Plant Cell, 23: 4221 - 4233 40. Xin, M., R. Yang, G. Li, H. Chen, J. Laurie, C. Ma, D. Wang. Y. Yao, B. Larkins, Q. Sun, R. Yadigari, X. Wang, and Z. Ni. 2013. Dynamic expression of imprinted genes associates whith maternally controlled nutrient allocation during maize endosperm development. The Plant Cell, 25(9): 3213 3227

41. Yadegari, R., and G. Drews. 2004. Female gametophyte development. Plant Cell, 16: S133 - S144

42. Yousif, M., and M. M. Elsahookie. 2007. Effect of xenia on traits of kernel and ear of maize subspecies crosses. TIJAS, 38(1): $113-$ 124

43. Zeamach, A., M. Kim, P. Silva, J. Rodrigues, and B. Dotson. 2010. Local DNA hypomethlation activates genes in rice endosperm. PNAS, 107: 18729 - 18734

44. Zeraati, M., D. Langley, P. SChofieid, A. Moye, R. Rouet, W. Hughes, T. Bryan, M. Dinger, and D. Christ. 2018. I-motif DNA structures are formed in the nuclei of human cells. Nature Chemistry, doi: 10.1038/S41557018-0046-3

45. Zhang, M., H. Zhao, S. Xie, J. Chen, Y. $\mathrm{Xu}, \mathrm{K}$. Wang, H. Zhao, H. Guan, X. Hu, Y. Jiao, W. Song, and J. Lai. 2011. Extensive, clustered parental imprinting of protein-coding and noncoding RNAs in developing maize endosperm. PNAS, 108(50): $20042-20047$

46. Zilberman, D., M. Gehring, R. Tran, T. Ballinger, and S. Henikoff. 2007. Genomewide analysis of Arabidopsis thaliana DNA methylation uncovers an interdependence between methylation and transcription. Nat. Genet., 39: 61 - 69

47. Zheng, X., L. Chen, H. Xia, H. Wei, Q. Lou, M. Li, and L. Luo. 2017. Transgenerational epimutations induced by multi-generation drought imposition mediate rice plants adaptation to drought condition. Sci. Rep., 7: 39843

48. Zhu, J. K. 2009. Active DNA demethylation mediated by DNA glycosylase. Annu. Rev. genet., 43:143 - 166. 